\title{
A WIENER TYPE THEOREM FOR DIRICHLET SERIES
}

\author{
ARTHUR GOODMAN AND D. J. NEWMAN
}

\begin{abstract}
A famous theorem of Wiener states the conditions under which the reciprocal of a function with an absolutely convergent Fourier series also has an absolutely convergent Fourier series.

We offer an elementary proof of the fact, first proven in $[\mathbf{2}]$, that if $F(s)$ has an absolutely convergent Dirichlet series then $1 / F(s)$ has an absolutely convergent Dirichlet series if and only if $|F(s)|$ is bounded away from zero in the closed right half-plane.
\end{abstract}

A famous theorem of Wiener states that if $f(x)$ has an absolutely convergent Fourier series and $f(x) \neq 0$, then $1 / f(x)$ also has an absolutely convergent Fourier series.

It is quite natural to ask whether or not an analogous result holds for Dirichlet series. In $[\mathbf{3}]$ E. Hewitt and J. H. Williamson considered the question for ordinary Dirichlet series and proved that a "reciprocal theorem" does hold in that special case. The question in its full generality was affirmatively answered by D. A. Edwards in [2]. However, both proofs rely rather heavily on the Gelfond theory of analytic functions on Banach algebras and the idea of maximal ideal spaces and, hence, are not particularly elementary.

We would therefore like to offer an entirely elementary proof of the general result (Theorem 1 below).

Let $F(s)$ be a Dirichlet series

$$
F(s)=a_{0}+\sum_{n=1}^{\infty} a_{n} e^{-\lambda_{n} s},
$$

where $s$ is a complex variable, $a_{0} \neq 0$ and the $\lambda_{n}$ are a sequence of distinct positive numbers. (In the case $\lambda_{n}=\log n$ the series is called an ordinary Dirichlet series.) Wherever it is notationally convenient to include $\lambda_{0}, \lambda_{0}=0$.

THEOREM 1. Let $F(s)$ be a Dirichlet series as defined in (1) and suppose $F(s)$ is absolutely convergent, i.e., $\sum_{n=0}^{\infty}\left|a_{n}\right|<\infty$. Then $1 / F(s)$ has an absolutely convergent Dirichlet series if and only if $|F(s)|>\delta>0$ for all $s$ with $\operatorname{Re} s \geq 0$.

Proof. The necessity of the condition is immediate, for if $1 / F(s)$ has an absolutely covergent Dirichlet series then it is bounded in Re $s \geq 0$, so $F(s)$ must be bounded away from zero in $\operatorname{Re} s \geq 0$.

In [5] D. J. Newman gave a simple proof of Wiener's "reciprocal theorem", and we follow a similar outline here to prove the sufficiency of the condition.

Received by the editors June 20, 1983.

1980 Mathematics Subject Classification. Primary 32A05; Secondary 41A10, 46B15.

Key words and phrases. Dirichlet series, Banach algebras, functions of several complex variables. positive basis, Kronecker's theorem, Reinhardt domain.

(C) 1984 American Mathematical Society $0002-9939 / 84 \$ 1.00+\$ .25$ per page 
Our basic outline is as follows. We take a partial sum of $F(s)$ and show that this partial sum, or Dirichlet polynomial as it is called, has as its reciprocal an absolutely convergent Dirichlet series. We then use the reciprocal of the partial sum to construct the reciprocal of $F(s)$.

We begin the sufficiency proof with several lemmas.

LEMMA 1. Given a finite set of positive numbers $\left\{\lambda_{1}, \ldots, \lambda_{M}\right\}$ there exists a positive basis for $\left\{\lambda_{1}, \ldots, \lambda_{M}\right\}$ over $Q$, i.e. there exists a linearly independent set $\left\langle\xi_{1}, \ldots, \xi_{r}\right\rangle$ of positive numbers such that each $\lambda_{i}$ is a linear combination of the $\xi_{i}$ with nonnegative rational coefficients.

PROOF. Given a finite set of numbers $\left\{\lambda_{1}, \ldots, \lambda_{M}\right\}$, the set of all linear combinations of $\lambda_{1}, \ldots, \lambda_{M}$ with rational coefficients is a vector space over $Q$, let us say of dimension $r$. Since $\lambda_{1}, \ldots, \lambda_{M}$ are positive numbers, the origin is clearly not contained in the positive span of $\lambda_{1}, \ldots, \lambda_{M}$, and so $\lambda_{1}, \ldots, \lambda_{M}$ must be contained in an open half-space through the origin. We may define this open half-space as the set of all rational combinations of $\lambda_{1}, \ldots, \lambda_{M}$ which, when viewed as real numbers, are positive.

Now consider the cone formed by $\lambda_{1}, \ldots, \lambda_{M}$. If we intersect this cone with a hyperplane parallel to the hyperplane which bounds the half-space, we get an $(r-1)$ dimensional polytope. Since the polytope is in an open half-space, we can enclose the polytope in an $(r-1)$-dimensional simplex also contained in the open half-space. In addition, we choose the simplex to have vertices with rational coordinates and join each of these $r$ vertices to the origin. We thus have our $r$ linearly independent vectors such that each $\lambda_{i}$ is contained in their nonnegative span. Let $\left\langle\xi_{1}, \ldots, \xi_{r}\right\rangle$ be those $r$ basis vectors. Since these basis vectors are in the open half-space defined above, they must be positive numbers, completing the proof of Lemma 1.

The next two lemmas deal with the behavior of polynomials in several complex variables.

LEMMA 2. Let $\alpha_{1}, \ldots, \alpha_{r}$ be positive numbers. If a polynomial $P\left(z_{1}, \ldots, z_{r}\right)$ has a zero at some point of the open unit polydisc, then it also has a zero at some point of the set $A=\left\{\left.\left(z_{1}, \ldots, z_{r}\right)|| z_{1}\right|^{\alpha_{1}}=\left|z_{2}\right|^{\alpha_{2}}=\cdots=\left|z_{r}\right|^{\alpha_{r}}<1\right\}$.

PROOF. The simple proof given here is due to John Wermer. We will need the following theorem from the theory of functions of several complex variables (see $[\mathbf{4}$, p. 35, Theorem 2.4.5]).

THEOREM A. Let $\Omega \subset C^{n}$ be a connected Rienhardt domain (i.e., $\left(z_{1}, \ldots, z_{n}\right) \in$ $\Omega$ implies $\left(e^{i \theta_{1}} z_{1}, \ldots, e^{i \theta_{n}} z_{n}\right) \in \Omega$ for all real $\left.\theta_{1}, \ldots, \theta_{n}\right)$ containing the origin, and let $f$ be holomorphic in $\Omega$. Then there exists a unique power series such that $f(z)=$ $\sum_{\alpha} a_{\alpha} z^{\alpha}$ where $\alpha=\left(\alpha_{1}, \ldots, \alpha_{n}\right)$ and $z=\left(z_{1}, \ldots, z_{n}\right)$ with normal convergence in $\Omega$.

We use this theorem to prove Lemma 2 as follows:

Let $Z(P)$ be the zero set of $P$ in $\left|z_{1}\right|<1, \ldots,\left|z_{r}\right|<1$ and let $\alpha_{1}, \ldots, \alpha_{r}$ be positive constants. Suppose $Z(P)$ fails to meet the set $A$ defined in Lemma 2 . Choose a neighborhood $\Omega$ of $A$ which is a Reinhardt domain such that $P \neq 0$ in $\Omega$. Then by Theorem $A$ there exists a power series which represents $1 / P$ in $\Omega$. Now since $(1,1, \ldots, 1) \in A$ and hence also in $\Omega$, this series will converge in the polydisc $\left|z_{k}\right|<1,1 \leq k \leq r$, and thereby provides an analytic continuation of $1 / \mathcal{P}$ to the 
polydisc. Therefore $P \neq 0$ in the polydisc. But this contradicts the assumption that $P$ has a zero in the polydisc. Consequently, we must conclude that if $P=0$ in the polydisc then $P$ must have a zero in $A$, which proves Lemma 2 .

Since our main theorem deals with functions of the form (1), let $D$ be the set of functions of the form (1) where the $\left\{\lambda_{i}\right\}$ can be any sequence of positive numbers. Then with the usual definition of addition and multiplication of series, $D$ is an algebra.

If, in addition, we set

$$
\|F\|=\sum_{n=0}^{\infty}\left|a_{n}\right|, \quad F \in D,
$$

$D$ becomes a Banach algebra. The completeness of $D$ follows immediately from the completeness of $l_{1}$. We note that as long as $\gamma_{i}>0$, a Dirichlet polynomial of the form

$$
Q(s)=b_{0}+\sum_{n=1}^{M} b_{n} e^{-\gamma_{n} s}, \quad b_{0} \neq 0
$$

is an element of $D$.

LEMMA 3. If $Q(s)$ is a Dirichlet polynomial of $K$ terms, say

$$
Q(s)=\sum_{n=1}^{K} b_{n} e^{-\gamma_{n} s}
$$

then

$$
\left\|Q^{m}\right\| \leq\left(\begin{array}{c}
m+K-1 \\
K-1
\end{array}\right)\left(\sup _{\operatorname{Re} s \geq 0}|Q(s)|\right)^{m} \quad \text { for } m=1,2,3, \ldots
$$

PROOF. By the Schwarz Inequality we have

$$
\|Q\|=\sum_{n=1}^{K}\left|b_{n}\right| \leq \sqrt{\sum_{n=1}^{K} 1^{2}} \cdot \sqrt{\sum_{n=1}^{K}\left|b_{n}\right|^{2}}
$$

But, as can readily be shown by direct computation,

$$
\sum_{n=1}^{K}\left|b_{n}\right|^{2}=\lim _{T \rightarrow \infty} \frac{1}{2 T} \int_{-T}^{T}|Q(i t)|^{2} d t
$$

Thus in (2) we have

$$
\|Q\| \leq \sqrt{\# \text { of terms in } Q} \cdot \sqrt{\lim _{T \rightarrow \infty} \frac{1}{2 T} \int_{-T}^{T}|Q(i t)|^{2} d t}
$$

so

$$
\|Q\| \leq \sqrt{\# \text { of terms in } Q} \cdot \sqrt{\sup _{t}|Q(i t)|^{2}}
$$

Thus

$$
\|Q\| \leq \sqrt{\# \text { of terms in } Q}\left(\sup _{t}|Q(i t)|\right)
$$


and

$$
\left\|Q^{m}\right\| \leq \sqrt{\# \text { of terms in } Q^{m}}\left(\sup _{t}|Q(i t)|\right)^{m} .
$$

We bound the number of terms in $Q^{m}$ as follows:

Since $Q$ has $K$ terms we write $Q=t_{1}+t_{2}+\cdots+t_{K}$; then

$$
Q^{m}=\sum_{\substack{e_{i} \geq 0 \\ e_{1}+e_{2}+\cdots+e_{K}=m}} t_{1}^{e_{1}} \cdot t_{2}^{e_{2}} \cdots t_{K}^{e_{K}}
$$

Thus the number of terms in $Q^{m}$ is bounded by the number of ways in which one can partition $m$ into $K$ parts. This is well known to be $\left(\begin{array}{c}m+K-1 \\ K-1\end{array}\right)$. So (3) becomes

$$
\left\|Q^{m}\right\| \leq\left(\begin{array}{c}
m+K-1 \\
K-1
\end{array}\right)\left(\sup _{t}|Q(i t)|\right)^{m},
$$

which completes the proof of Lemma 3 .

With these lemmas in hand we return to the proof of the sufficiency of the condition in Theorem 1.

Suppose $F(s) \in D$ and $|F(s)|>\delta>0$ for $\operatorname{Re} s \geq 0$. Without loss of generality we assume $\delta=1$, so we have

$$
|F(s)|>1 \text {. }
$$

By the hypothesis of our theorem, $F(s)$ is absolutely convergent, so we can choose $P(s)$ a high enough partial sum of $F(s)$ such that

$$
\|P(s)-F(s)\| \leq \frac{1}{3}, \quad \text { say } P(s)=a_{0}+\sum_{n=1}^{M} a_{n} e^{-\lambda_{n} s} .
$$

Since the $\lambda_{1}, \ldots, \lambda_{M}$ are positive, Lemma 1 assures us the existence of a positive set $\left\langle\xi_{1}, \ldots, \xi_{r}\right\rangle$, linearly independent over $Q$, such that

$$
\lambda_{n}=e_{1}(n) \xi_{1}+e_{2}(n) \xi_{2}+\cdots+e_{r}(n) \xi_{r},
$$

where $e_{j}(n)$ are nonnegative rationals for $1 \leq j \leq r, 1 \leq n \leq M$. We also define $e_{i}(0)=0$.

Now consider the following mapping $T$, defined to be linear and multiplicative.

$$
T: e^{-\lambda_{n} s} \rightarrow z_{1}^{e_{1}(n)} z_{2}^{e_{2}(n)} \cdots z_{r}^{e_{r}(n)} \text { for } 0 \leq n \leq M .
$$

If we take as $D^{*}$ the space of formal series of the form

$$
g\left(z_{1}, \ldots, z_{k}\right)=\sum_{n=0}^{\infty} c_{n} z_{1}^{t_{1}} \cdots z_{k}^{t_{k}}
$$

where the $t_{i}$ are nonnegative rational numbers, and if we define

$$
\|g\|_{D^{*}}=\sum_{n=0}^{\infty}\left|c_{n}\right|
$$

then $T$ induces an isometry between $D$ and $D^{*}$.

The first step in the proof consists of showing that Theorem 1 holds for $P(s)$ i.c., that $1 / P(s)$ has an absolutely convergent Dirichlet series. 
We do this by examining the polynomial in several complex variables which is associated by $T$ with $P(L s)$, where $L=$ least common multiple of the denominators of $e_{j}(n), 1 \leq j \leq r ; 1 \leq n \leq M$. That is, let

$$
T: P(L s) \rightarrow P\left(z_{1}, \ldots, z_{r}\right)=\sum_{n=0}^{M} a_{n} z_{1}^{L e_{1}(n)} \cdots z_{r}^{L e_{r}(n)} .
$$

From the definition of the norm in $D$ we clearly have $|P-F| \leq\|P-F\|$, so from (4) and (5) we get

$$
|P(s)| \geq 2 / 3 \text {. }
$$

If we can show that $\left|\mathcal{P}\left(z_{1}, \ldots, z_{r}\right)\right|>c>0$ for $\left|z_{i}\right| \leq 1,1 \leq i \leq r$, then $1 / P\left(z_{1}, \ldots, z_{r}\right)$ is an analytic function in a region which contains the unit polydisc, so its power series is absolutely convergent in the unit polydisc. But since $T$ is an isometry and $P(L s)$ and $P(s)$ have the same coefficients, $1 / P(s)$ has an absolutely convergent Dirichlet series. The fact that $\left|P\left(z_{1}, \ldots, z_{r}\right)\right|>c>0$ is an immediate consequence of $(6)$ and the following lemma.

LEMMA 4. The range of $P\left(z_{1}, \ldots, z_{r}\right)$ in $\left|z_{i}\right| \leq 1,1 \leq i \leq r$, is identical to the closure of the range of $P(s)$ in $\operatorname{Re} s \geq 0$.

PROOF OF LEMMA 4

(7) At points of the form $z_{k}^{\prime}=e^{-\xi_{k} s / L}$ we have

$$
\begin{aligned}
P\left(z_{1}^{\prime}, \ldots, z_{r}^{\prime}\right) & =\sum_{n=0}^{M} a_{n} e^{\left(-\xi_{1} s / L\right) \cdot L e_{1}(n)} \cdots e^{\left(-\xi_{r} s / L\right) \cdot L e_{r}(n)} \\
& =\sum_{n=0}^{M} a_{n} e^{-\left(e_{1}(n) \xi_{1}+\cdots+e_{r}(n) \xi_{r}\right)}=\sum_{n=0}^{M} a_{n} e^{-\lambda_{n} s}=P(s) .
\end{aligned}
$$

(8) Thus $P\left(z_{1}^{\prime}, \ldots, z_{r}^{\prime}\right)=P(s)$.

We prove the lemma by showing that given any $\varepsilon>0$ and any point $\left(z_{1}, \ldots, z_{r}\right)$ with $\left|z_{i}\right| \leq 1$, we can find $z_{k}^{\prime}$ as defined in (7), with $\operatorname{Re} s \geq 0$ such that

$$
\left|\mathcal{P}\left(z_{1}, \ldots, z_{r}\right)-\mathcal{P}\left(z_{1}^{\prime}, \ldots, z_{r}^{\prime}\right)\right|<\varepsilon .
$$

(9) will follow by continuity if, given any $\delta>0$ and any point $\left(z_{1}, \ldots, z_{r}\right)$ with $\left|z_{i}\right| \leq 1$, we can find $z_{k}^{\prime}$ as defined in (7) with Re $s \geq 0$ such that

$$
\left|z_{k}-z_{k}^{\prime}\right|<\delta \text { for } 1 \leq k \leq r .
$$

We establish (10) with the aid of Kronecker's Theorem.

If $\left|z_{k}\right|=1$ for $1 \leq k \leq r$ then $z_{k}=e^{2 \pi i \alpha_{k}}$ where the $\alpha_{k}$ are real numbers.

Let $\xi_{r+1}$ be any positive real number which is not in the rational span of $\left\langle\xi_{1}, \ldots, \xi_{r}\right\rangle$. If we take $\theta_{k}=-\xi_{k} /\left(L \cdot \xi_{r+1}\right)$ for $1 \leq k \leq r$, it is easily shown that the set $\left\{1, \theta_{1}, \ldots, \theta_{r}\right\}$ is linearly independent. Therefore we can apply Kronecker's Theorem and get

$$
\left|e^{2 \pi i n \theta_{k}}-e^{2 \pi i \alpha_{k}}\right|<\delta .
$$

But $e^{2 \pi i n \theta_{k}}=e^{-\xi_{k} s / L}$, where $s=2 \pi n i / \xi_{r+1}$, so $e^{2 \pi i n \theta_{k}}$ is a $z_{k}^{\prime}$ and (11) becomes (10). 
If $\left|z_{k}\right|<1$ for $1 \leq k \leq r$ then, by virtue of Lemma 2 with $\alpha_{k}=1 / \xi_{k}$, we can find a point $\left(w_{1}, \ldots, w_{r}\right)$ with $\left|w_{i}\right|<1$ such that

$$
\left|w_{1}\right|^{1 / \xi_{1}}=\left|w_{2}\right|^{1 / \xi_{2}}=\cdots=\left|w_{r}\right|^{1 / \xi_{r}} \text { and } P\left(z_{1}, \ldots, z_{r}\right)=\mathcal{P}\left(w_{1}, \ldots, w_{r}\right) .
$$

Since $\left|w_{k}\right|<1$ for $1 \leq k \leq r$ we have $\left|w_{k}\right|^{1 / \xi_{k}}=e^{-a}, a>0,1 \leq k \leq r$ and, hence,

$$
\left|w_{k}\right|=e^{-a \xi_{k}}, \quad a>0,1 \leq k \leq r .
$$

Also since $\left|w_{k}\right|<1$, we have $w_{k}=\rho_{k} e^{2 \pi i \alpha_{k}}$, where $\alpha_{k}$ are arbitrary real numbers, and, by (12), $\rho_{k}=e^{-a \xi_{k}}$. Now choose

$$
z_{k}^{\prime}=e^{\left(-a \xi_{k}\right.} e^{\left.2 \pi i n / L \xi_{r+1}\right) \cdot \xi_{k}}=e^{-\xi_{k} s / L} \quad \text { where } s=L a-2 \pi n i / \xi_{r+1} .
$$

Since $a>0$ we have $\operatorname{Re} s \geq 0$ as required. Applying Kronecker's Theorem as before establishes (10).

In the case where $\left(z_{1}, \ldots, z_{r}\right)$ has mixed coordinates, i.e., some inside and some on the boundary of the unit polydisc, then clearly a combination of the above arguments produces the required $z_{k}^{\prime}$.

We have thus far established that Theorem 1 applies to $P(s)$, so $1 / P(s)$ has an absolutely convergent Dirichlet series. So we can find a $K$ such that the $K$ th partial sum of $1 / P(s)$, let us call it $Q(s)$, satisfies

$$
\|1 / P(s)-Q(s)\|<\frac{1}{2} \text {. }
$$

Let $\Delta=1 / P(s)-Q(s)$ so that $\|\Delta\|<1 / 2$. Now consider the sum

$$
L(s)=\sum_{n=1}^{\infty}(P(s)-F(s))^{n-1} /(P(s))^{n} .
$$

We prove that $L$ converges in norm to $1 / F$ and, hence, $1 / F$ has an absolutely convergent Dirichlet series. From (13) we have that $Q+\Delta=1 / P$, while from (5), $\|P-F\| \leq 1 / 3$. Thus

$$
\left\|\frac{(P-F)^{n-1}}{P^{n}}\right\| \leq\left\|(P-F)^{n-1}\right\| \cdot\left\|\frac{1}{P^{n}}\right\|<\frac{1}{3^{n-1}} \cdot\left\|(Q+\Delta)^{n}\right\| .
$$

Again from (13), $\|\Delta\|<1 / 2$, so applying the Binomial Theorem and triangle inequality in (14) gives

$$
\left\|(Q+\Delta)^{n}\right\| \leq\left\|Q^{n}\right\|+\left(\begin{array}{c}
n \\
1
\end{array}\right) \cdot \frac{1}{2}\left\|Q^{n-1}\right\|+\cdots+\left(\begin{array}{c}
n \\
m
\end{array}\right) \frac{1}{2^{n-m}}\left\|Q^{m}\right\|+\cdots+\frac{1}{2^{n}}
$$

From (6) we get $|1 / P| \leq 3 / 2$, which, together with (13), gives

$$
|Q|=|1 / P+\Delta| \leq 2 .
$$

Applying Lemma 3, together with (16), yields

$$
\|Q\|^{m} \leq \sqrt{\left(\begin{array}{c}
m+K-1 \\
K-1
\end{array}\right)} \cdot 2^{m} \quad \text { for } 1 \leq m \leq n .
$$

But since $1 \leq m \leq n$

$$
\left(\begin{array}{c}
m+K-1 \\
K-1
\end{array}\right) \leq\left(\begin{array}{c}
n+K-1 \\
K-1
\end{array}\right)
$$


and we have

$$
\|Q\|^{m} \leq \sqrt{\left(\begin{array}{c}
n+K-1 \\
K-1
\end{array}\right)} \cdot 2^{m} \quad \text { for } 1 \leq m \leq n .
$$

Using this last result in (15) yields

$$
\begin{aligned}
\left\|(Q+\Delta)^{n}\right\| \leq \sqrt{\left(\begin{array}{c}
n+K-1 \\
K-1
\end{array}\right)}\left(2^{n}+n \cdot \frac{1}{2} \cdot 2^{n-1}\right. & \\
& \left.+\cdots+\left(\begin{array}{c}
n \\
m
\end{array}\right)\left(\frac{1}{2}\right)^{n-m} 2^{m}+\cdots+\left(\frac{1}{2}\right)^{n}\right),
\end{aligned}
$$

so

$$
\left\|(Q+\Delta)^{n}\right\| \leq \sqrt{\left(\begin{array}{c}
n+K-1 \\
K-1
\end{array}\right)}\left(\frac{5}{2}\right)^{n}
$$

Substituting this estimate into (14) gives

$$
\left\|\frac{(P-F)^{n-1}}{P^{n}}\right\| \leq \sqrt{\left(\begin{array}{c}
n+K-1 \\
K-1
\end{array}\right)}\left(\frac{5}{2}\right) / 3^{n-1}=3 \sqrt{\left(\begin{array}{c}
n+K-1 \\
K-1
\end{array}\right)}\left(\frac{5}{6}\right)^{n},
$$

and we see that $L(s)$ converges in norm by the ratio test.

However if a series $1+x+x^{2}+\cdots+x^{n}+\cdots$ converges in norm, it must converge to $1 /(1-x)$. This follows the fact that

$$
\lim _{n \rightarrow \infty}(1-x)\left(1+x+x^{2}+\cdots+x^{n}\right)=\lim _{n \rightarrow \infty}\left(1-x^{n-1}\right),
$$

so it is both necessary and sufficient to have $\lim _{n \rightarrow \infty} x^{n}=0$. But the convergence of the series insures that $\lim _{n \rightarrow \infty} x^{n}=0$.

Consequently $L(s)$ converges in norm to

$$
\frac{1}{P} \cdot \frac{1}{1-(P-F) / P}=\frac{1}{F}
$$

and this concludes the proof of Theorem 1 .

\section{BIBLIOGRAPHY}

1. G. H. Hardy and M. Riesz, The general theory of Dirichlet series, Cambridge Univ. Press, 1952.

2. D. A. Edwards, On absolutely convergent Dirichlet series, Proc. Amer. Math. Soc. 8 (1957), 1067-1074.

3. Edwin Hewitt and J. H. Williamson, Note on absolutely convergent Dirichlet series, Proc. Amer. Math. Soc. 8 (1957), 863-868.

4. L. Hörmander, An introduction to complex analysis in several complex variables, Van Nostrand, 1966.

5. D. J. Newman, A simple proof of Wiener's 1/f theorem, Proc. Amer. Math. Soc. 48 (1975), 264-265.

Department of Mathematics, Queens College, City University of NeW YORK, FLUSHING, NEW YORK 11367

Department of Mathematics, Temple University, Philadelphia, PennsylvaNIA 19122 